Classification of breast masses by ultrasonic Nakagami imaging: a feasibility study

This article has been downloaded from IOPscience. Please scroll down to see the full text article.

2008 Phys. Med. Biol. 536027

(http://iopscience.iop.org/0031-9155/53/21/009)

The Table of Contents and more related content is available

Download details:

IP Address: 140.112.113.225

The article was downloaded on 23/12/2008 at 04:42

Please note that terms and conditions apply. 


\title{
Classification of breast masses by ultrasonic Nakagami imaging: a feasibility study
}

\author{
Po-Hsiang Tsui ${ }^{1}$, Chih-Kuang Yeh ${ }^{2}$, Chien-Cheng Chang ${ }^{1,3,4}$ \\ and Yin-Yin Liao ${ }^{2}$ \\ ${ }^{1}$ Division of Mechanics, Research Center for Applied Sciences, Academia Sinica, 128, \\ Section 2, Academia Road, Nankang, Taipei 11529, Taiwan, Republic of China \\ 2 Department of Biomedical Engineering and Environmental Sciences, National Tsing Hua \\ University, Hsinchu, Taiwan, Republic of China \\ ${ }^{3}$ Institute of Applied Mechanics, National Taiwan University, Taipei, Taiwan, Republic of China \\ E-mail: mechang@gate.sinica.edu.tw (Chien-Cheng Chang) and ckyeh@mx.nthu.edu.tw \\ (Chih-Kuang Yeh)
}

Received 21 April 2008, in final form 21 August 2008

Published 3 October 2008

Online at stacks.iop.org/PMB/53/6027

\begin{abstract}
Ultrasound is an important clinical tool in noninvasive diagnoses of breast cancer. The Nakagami statistical parameter estimated from the ultrasonic backscattered envelope has been demonstrated to be useful in complementing conventional B-mode scans when classifying breast masses. However, the shadowing effect caused by certain high-attenuation tumors in the B-mode image makes the tumor contour unclear, and thus it is more difficult to choose an appropriate region of interest from which to collect tumor data for estimating the Nakagami parameter. This study explored the feasibility of using the Nakagami parametric image to overcome the shadowing effect for visualizing the properties of breast masses. Experiments were performed on a breastmimicking phantom and on some typical clinical cases for cysts, fat and tumors (fibroadenoma) $(n=18)$ in order to explore the performance of the Nakagami image under ideal and practical conditions. The experimental results showed that the Nakagami image pixels (i.e. the local Nakagami parameter) in the cyst, tumor and fat are $0.21 \pm 0.01,0.65 \pm 0.05$ and $0.98 \pm 0.07$, respectively, for six independent phantom measurements, and $0.14 \pm 0.03,0.67 \pm 0.11$ and $0.89 \pm$ 0.08 , respectively, for clinical experiments. This suggests that the Nakagami image is able to classify various breast masses $(p<0.005)$ although the clinical results from tumors of different cases have a larger variance that may be caused by the complexity of real breast tissues. In particular, unlike the B-mode image, the Nakagami image is not subject to significant shadowing effects, making it useful to complement the B-mode image to describe the tumor contour for
\end{abstract}

4 Author to whom correspondence should be addressed. 
identifying the tumor-related region when the shadowing effect is stronger or a low system gain is used.

(Some figures in this article are in colour only in the electronic version)

\section{Introduction}

Breast cancer is a public health problem worldwide. X-ray mammography is the most important imaging methodology for diagnosing breast cancer due to its high sensitivity and resolution aiding early detection (Moore 2001). However, mammography has some limitations in clinical use. First, the ionizing nature of x-ray radiation limits the frequency and dosage of its application, and $\mathrm{x}$-ray mammography does not reveal soft tissues. Second, the sensitivity of x-ray mammography is lower for dense breasts (Kolb et al 1998, Ma et al 1992) due to their larger attenuation, and hence the high density of breasts of women younger than 40 years (especially Asian women) makes it challenging to image them by using X-ray mammography (Kubik-Huch 2006).

The use of x-ray mammography in screening young breasts is often complemented with ultrasound imaging, which has become an important imaging tool in the noninvasive diagnosis of breast cancer (Alizad et al 2006). Ultrasound imaging has many advantages over mammography and other imaging methods, such as magnetic resonance imaging and optical imaging (Moore 2001), including noninvasiveness, nonionizing radiation, ease of use, short examination time and comparatively low cost, making it very convenient and suitable for routine and frequent breast screening. An ultrasound image can provide clear visualization of soft tissues, which do not show up well in x-ray images. Furthermore, it can be used to differentiate between cysts and solid masses (Zonderland 2000, Jackson 1990) and to detect masses that are not visible in X-ray mammography of dense breasts (Jackson 1990). Nevertheless, current breast ultrasound methods are still subject to some limitations. In some cases, the ultrasound image cannot be used to classify different breast masses, because the brightness and other characteristics of ultrasonic B-mode scans are dependent on many factors including the system gain, dynamic range, and signal and image processing techniques (Shung et al 1992), making the classification of the properties of breast masses largely dependent on the skill of the operator, leading to subjective evaluations.

Many studies have shown that the quantitative analysis of ultrasound images is useful for classifying breast masses. The Nakagami statistical distribution, initially proposed to describe the statistics of radar echoes (Holfman 1960), and the associated Nakagami parameter have recently received considerable attention due to the fact that the Nakagami distribution has been shown to be a general model for all scattering conditions encountered in medical ultrasound (Shankar 2000). Moreover, the Nakagami parameter can be used to distinguish different scattering conditions and scatterer concentrations in a scattering medium (Shankar 2000, Tsui and Wang 2004). Several studies have shown that the Nakagami parameter estimated from the ultrasonic backscattered signals can be used to assist conventional B-mode scanning when classifying breast masses (Shankar et al 2001, 2003a, Dumane et al 2002a). Also, combining the Nakagami parameter with additional techniques such as frequency diversity and compounding (Shankar et al 2002, Dumane et al 2002b), a multiparameter approach (Shankar et al 2003b) and the Nakagami compounding distribution (Shankar et al 2005) can also improve the effectiveness of using the Nakagami parameter to classify breast masses. 
Describing the properties of breast masses using a parameter-based approach is not without any disadvantages. First, a single parameter cannot be used to characterize the breast mass locally. Second, a tumor usually exhibits the so-called shadowing effects in B-mode images (Drukker et al 2003, Whatmough et al 2006), making the mass contour unclear and thereby increasing the difficulty of segmenting a breast mass. For some cases with strong shadowing effects or improper imaging settings, the shadow area may look like a part of the tumor-related region, and therefore it would become more difficult to tell the true tumor region. Under this condition, if we need all backscattering data in the tumor for analyses, we may easily choose an inappropriate region of interest to introduce bias when estimating the Nakagami parameter of the mass. Using the ultrasonic parametric image to visualize the breast properties might be a feasible method for overcoming the above problems. The parametric image allows local characterization of tissues, and more importantly is typically less dependent on the amplitude of received echoes and hence can avoid the shadowing effects and thereby improve the detection of the tumor-related region.

In the present work, we explored the feasibility of classifying different breast masses by using the parametric image based on the Nakagami parameter (i.e. the Nakagami image). Note that there are many types of ultrasound parametric images, such as integrated backscatter (Bridal et al 1997) and attenuation images (Bevan and Sherar 2001a, 2001b). The Nakagami image belongs to the statistical parameter image, which is responsible for analyzing the backscattering statistics. The concept of Nakagami imaging originated from the suggestion of Shankar (2002), which prompted some preliminary studies (Kolář et al 2004, Davignon et al 2005). We have previously proposed a standard procedure for forming the Nakagami image (Tsui and Chang 2007), and confirmed its usefulness in visualizing local scatterer concentrations in biological tissues (Tsui et al 2007).

This paper is organized as follows. Section 2 introduces the theoretical background. Section 3 shows materials and methods, section 4 shows experimental results obtained from a breast phantom and some clinical data for discussing the performance of the Nakagami image to classify breast masses under ideal and practical conditions and, finally, section 5 concludes with some important findings and remarks on the possible role of the Nakagami image in breast ultrasound imaging.

\section{Theoretical background}

\subsection{Nakagami distribution}

The probability density function (pdf) of the ultrasonic backscattered envelope $R$ under the Nakagami statistical model is given by

$$
f(r)=\frac{2 m^{m} r^{2 m-1}}{\Gamma(m) \Omega^{m}} \exp \left(-\frac{m}{\Omega} r^{2}\right) U(r)
$$

where $\Gamma(\cdot)$ and $U(\cdot)$ are the gamma function and the unit step function, respectively. Let $E(\cdot)$ denote the statistical mean; then the scaling parameter $\Omega$ and the Nakagami parameter $m$ associated with the Nakagami distribution can be respectively obtained from

$$
\Omega=E\left(R^{2}\right)
$$

and

$$
m=\frac{\left[E\left(R^{2}\right)\right]^{2}}{E\left[R^{2}-E\left(R^{2}\right)\right]^{2}} .
$$

The Nakagami parameter $m$ is a shape parameter determined by the pdf of the backscattered envelope. As $m$ varies from 0 to 1 , the envelope statistics changes from a 


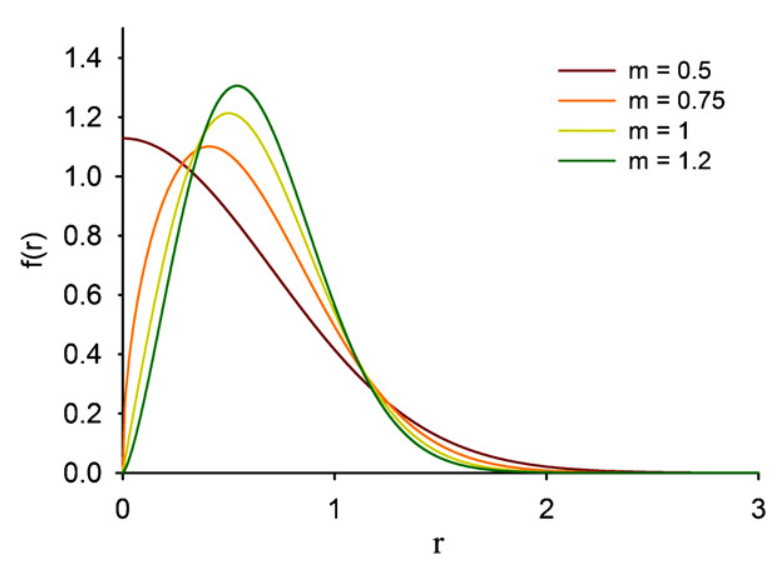

Figure 1. Nakagami distributions for different Nakagami parameters.

pre-Rayleigh to a Rayleigh distribution, and the backscattered statistics conform to postRayleigh distributions if $m$ is larger than 1, as shown in figure 1. This property makes the Nakagami distribution a good general model for ultrasonic backscattering.

\subsection{Nakagami imaging}

Nakagami imaging is detailed elsewhere (Tsui and Chang 2007). In brief, the Nakagami image is based on the Nakagami parameter map, which is constructed by using a square sliding window to process the envelope image (without logarithmic compression). This involves two main steps, as follows.

(1) A square window within the envelope image is used to collect the local backscattered envelopes for estimating the local Nakagami parameter $m_{\mathrm{w}}$, which is assigned as the new pixel located in the center of the window.

(2) Step 1 is repeated with the window moving throughout the entire envelope image in steps of 1 pixel, which yields the Nakagami image as the map of $m_{\mathrm{w}}$ values.

Note that the window size determines the resolution of the Nakagami image, with the use of a smaller window improving the resolution. However, a small window has fewer envelope data points, which can lead to unstable estimates of $m_{\mathrm{w}}$. Therefore, prior to constructing the Nakagami image, the optimal window size that simultaneously satisfies both stable estimations of $m_{\mathrm{w}}$ and an acceptable imaging resolution needs to be determined using the following steps.

(1) A reference homogeneous medium is scanned to obtain its envelope image. Each envelope signal of the image scan line is used to calculate the Nakagami parameter $m$. The average Nakagami parameter $\bar{m}$ is calculated by averaging all $m$ parameters. Under the condition that the length of the envelope signal is long enough to satisfy the stable estimation of $m, \bar{m}$ can be treated as an indicator of the global backscattered statistics of the scanning region.

(2) Each $m_{\mathrm{w}}$ in the scanning region and the average value $\bar{m}_{\mathrm{w}}$ are subsequently estimated using progressively larger sliding windows. Assuming that the window is large enough (but not too large) to satisfy the stable estimation of the parameter $m_{\mathrm{w}}, \bar{m}_{\mathrm{w}}$ should approach $\bar{m}$ to reflect the identical global backscattered statistics. Consequently, the optimal window size is determined once $\bar{m}_{\mathrm{w}}=\bar{m}$. 
Table 1. Characteristics of the materials in the breast phantom

\begin{tabular}{lllllc}
\hline & $\begin{array}{l}\text { Radius } \\
\text { Material }\end{array}$ & $\begin{array}{l}\text { Sound velocity } \\
\text { at } 5 \mathrm{MHz} \text { and }\end{array}$ & $\begin{array}{l}\text { Density } \\
\left.22{ }^{\circ}{\mathrm{C}\left(\mathrm{m} \mathrm{s}^{-1}\right)}^{-3}\right)\end{array}$ & $\begin{array}{l}\text { Attenuation } \\
\text { coefficient at } \\
5 \mathrm{MHz}\left(\mathrm{gB} \mathrm{cm}^{-1}\right)\end{array}$ & $\begin{array}{l}\text { Contrast relative } \\
\text { to glandular } \\
\text { tissue }(\mathrm{dB})\end{array}$ \\
\hline $\begin{array}{l}\text { Glandular } \\
\text { tissue }\end{array}$ & $\mathrm{N} / \mathrm{A}$ & 1522 & 1.03 & 2.74 & - \\
Fat & 4 & 1464 & 0.94 & 2.21 & -14 \\
Cyst & 4 & 1570 & 1.02 & 0.78 & $<-14$ \\
$\begin{array}{l}\text { Tumor } \\
\text { Irregular }\end{array}$ & 4 & 1547 & 1.10 & 7.36 & -12 \\
tumor & $\mathrm{N} / \mathrm{A}$ & 1553 & 1.07 & 4.26 & -10 \\
\hline
\end{tabular}

Based on the conclusion in our previous study (Tsui and Chang 2007), the appropriate sliding window used to construct the Nakagami image is suggested as a square with a side length equal to three times the pulse length of the incident ultrasound. A pseudocolor scale was applied to clearly reveal the information in the Nakagami image. Values of $m_{\mathrm{w}}$ smaller than 1 were assigned blue shading that changed from dark to light with an increasing value, representing the backscattered envelope that conformed to various pre-Rayleigh statistics. Pixels with $m_{\mathrm{w}}$ equal to 1 were shaded white to indicate a Rayleigh distribution, and those larger than 1 were assigned red shading from dark to light with the increasing value, indicating backscattered statistics with various degrees of a post-Rayleigh distribution.

\section{Materials and methods}

In this feasibility study, we first used a custom-made phantom to mimic different breast masses. This breast phantom was constructed by Professor Ernest L Madsen (Department of Medical Physics, University of Wisconsin-Madison), and has previously been shown to be capable of simulating the properties of different breast masses (Huang and Li 2004, 2005, Glide et al 2007). The phantom made of uniform materials includes fat, a cyst, a tumor and an irregular tumor. The radii of the cyst, tumor and fat are $4 \mathrm{~mm}$. The irregular tumor has no fixed size and shape; these are dependent on the angle and region of ultrasound scanning. The characteristics of the materials in the phantom are listed in table 1 . The experimental setup is shown in figure 2 . The ultrasound radio-frequency (RF) signals from the breast phantom are acquired using a commercial ultrasound scanner (Model 2000, Terason, Burlington, MA, USA), with the raw RF data digitized at a sampling rate of $30 \mathrm{MHz}$. The applied probe is a wideband linear array with a central frequency of $7.5 \mathrm{MHz}$ and 128 elements (Model 10L5, Terason). In order to estimate the pulse length of the incident wave, two-way pulse-echo testing of the ultrasound transducer was carried out. We placed both the transducer and a steel reflector in a water bath, and adjusted the distance between the transducer and reflector to be the focal length of the transducer (focal length is adjustable for the Terason system). Then, we acquired the echo signal from the reflector for estimating the pulse length. It showed that the pulse length was approximately $0.7 \mathrm{~mm}$.

Ultrasound gel was applied between the transducer and the surface of the phantom, and different cross-sectional areas corresponding to the fat, cyst, tumor and the irregular tumor were scanned six times each. Each image acquisition protocol involved obtaining a total of 128 image scan lines of backscattered echoes. Each scan line was then demodulated using the Hilbert transform to obtain the envelope image, and the B-mode image formed based on 


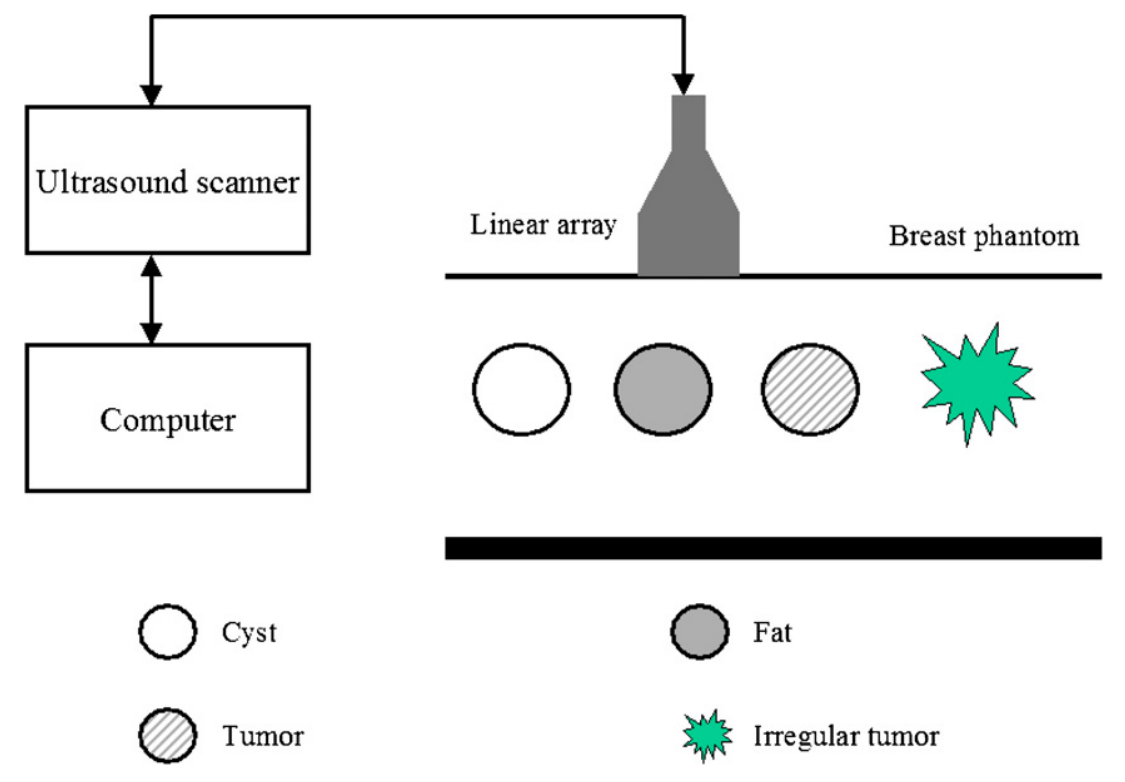

Figure 2. Experimental setup.

the logarithm-compressed envelope image at a dynamic range of $40 \mathrm{~dB}$. The Nakagami image corresponding to each B-mode image was formed according to the algorithmic procedure described in section 2. We constructed the Nakagami images of the breast phantom using a square window with a size of $2.1 \times 2.1 \mathrm{~mm}^{2}$. We also collected some typical breast mass data from the Mackay Memorial Hospital, Hsinchu, Taiwan, to explore the clinical usefulness of Nakagami images of the breast. The patients signed agreement forms for participating in the experiments. A sonographer performed the ultrasound scanning and a doctor identified breast masses. The data obtained comprised three cases: cyst $(n=4)$, fibroadenoma $(n=8)$ and fat tissue $(n=6)$.

\section{Results and discussion}

\subsection{Results on a phantom}

Figures 3(a) and (b) show the typical B-mode image and the corresponding Nakagami image of a cyst, respectively. Figure 3(c) shows the histogram of the Nakagami image pixels (i.e. local Nakagami parameter $m_{\mathrm{w}}$ ) in the cyst. The cyst region of the Nakagami image was associated with extremely dark-blue shading, corresponding to very small $m_{\mathrm{w}}$ parameters ranging from 0.05 to 0.35 . We further performed image scanning six times (at different cross-sectional areas), and calculated the average and standard deviation of $\bar{m}_{\mathrm{w}}$ values obtained from each independent scan $\left(\mu_{\bar{m}_{\mathrm{w}}} \pm \mathrm{SD}\right)$. This yielded $\mu_{\bar{m}_{\mathrm{w}}} \pm \mathrm{SD}=0.21 \pm 0.01$, indicating that the global statistical distribution of the backscattered envelopes from the cyst conformed to preRayleigh statistics. This was due to the fact that there were few scatterers in the cyst region to contribute backscattered echoes. However, the cyst region was slightly larger in the Nakagami image than in the B-mode image, which was due to the use of a square window with a side length equal to three times the ultrasound pulse length to construct the Nakagami image, which results in worse image resolution. 

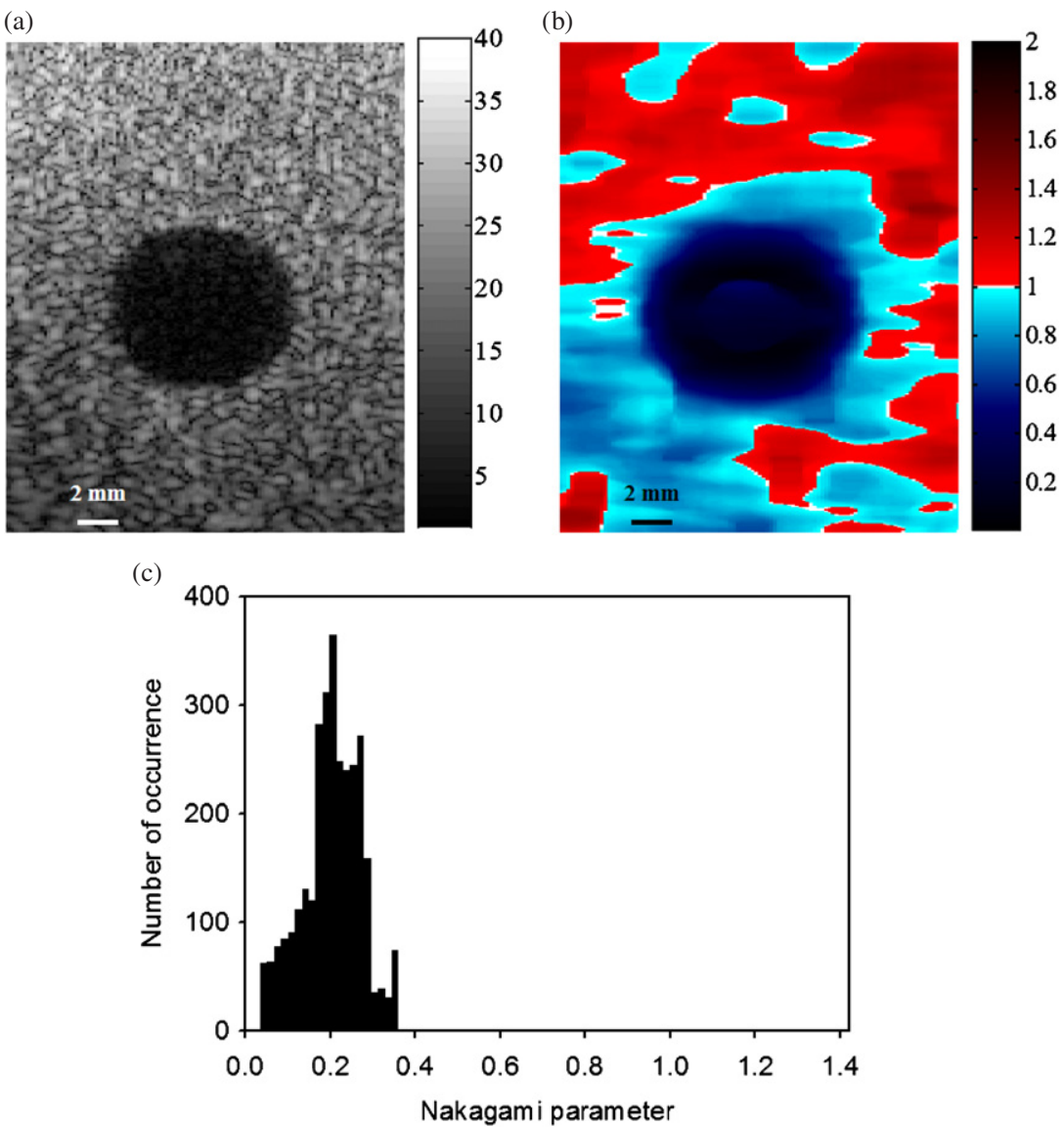

Figure 3. Typical B-mode image (a), Nakagami image (b) and the histogram of the Nakagami image pixels (c) for the cyst.

Figures 4(a) and (b) show the B-mode and Nakagami images of fat, respectively. Figure 4(c) shows the $m_{\mathrm{w}}$ histogram in the fat. The shading of the Nakagami image for the fat differed from that for the cyst. The fat region was associated with both red and blue shading corresponding to the $m_{\mathrm{w}}$ values with a distribution from 0.9 to 1.4 , indicating that some of the statistics of the local backscattered envelope conformed to a post-Rayleigh distribution, with the remainder conforming to a pre-Rayleigh distribution. The $\mu_{\bar{m}_{\mathrm{w}}} \pm$ SD value in the fat was $0.98 \pm 0.07$, indicating global backscattered statistics conforming to a Rayleigh distribution. This implies that the Nakagami image identified fat as a region containing a large number of randomly distributed scatterers (i.e. a homogeneous medium).

Figures 5(a) and (b) show the B-mode and Nakagami images of the tumor, respectively. Figure 5(c) shows the $m_{\mathrm{w}}$ histogram in the tumor. The shading in the Nakagami image for a tumor differs from those for a cyst and fat. The tumor region was associated with blue shading corresponding to the $m_{\mathrm{w}}$ parameters ranging from 0.45 to 0.7 . More precisely, $\mu_{\bar{m}_{\mathrm{w}}} \pm \mathrm{SD}$ was approximately $0.65 \pm 0.05$ for the tumor, indicating envelope statistics that conformed more to a pre-Rayleigh distribution than it did in the case of the cyst. Note that there was an apparent shadowing effect in the B-mode image. This is because the tumor has larger acoustic attenuation, as listed in table 1. In principle, the Nakagami image should be 


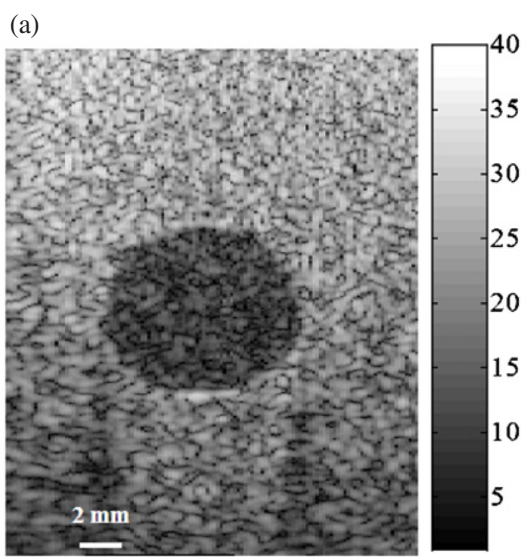

(b)

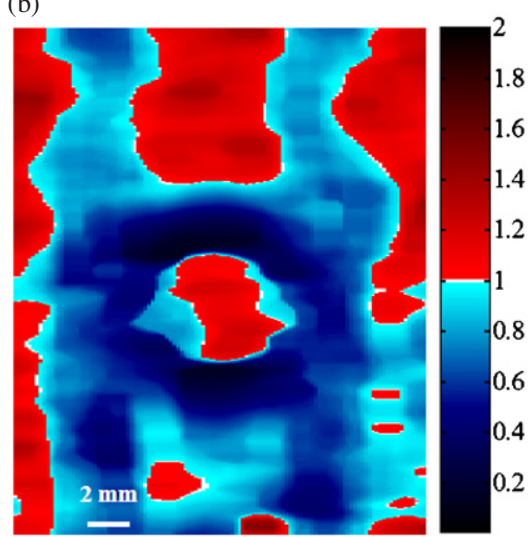

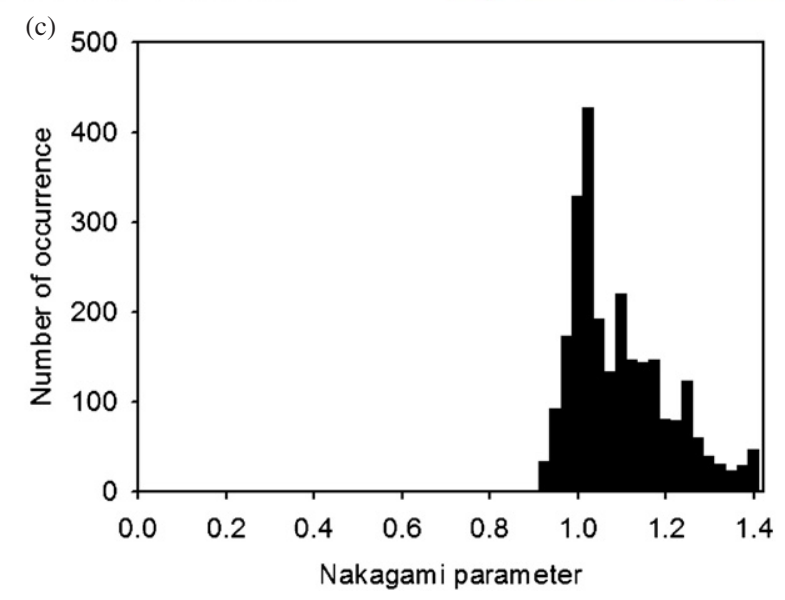

Figure 4. Typical B-mode image (a), Nakagami image (b) and the histogram of the Nakagami image pixels (c) for the fat.

less affected by the attenuation effect because the Nakagami parameter is mainly dependent on the statistical distribution of the backscattered envelope and less influenced by the echo amplitude. This can be demonstrated by figure 5(b) and by comparing representative vertical image profiles (i.e. scan lines) of the B-mode and Nakagami images, as shown in figure 6 . The averages of the relative envelope intensities on and beneath the tumor were 0.33 and 0.04 , respectively. Assuming that the system noise was a constant level throughout the scanning region, it can be considered that the signal-to-noise ratio (SNR) of the echo decreased by $87 \%$ when ultrasound propagated through the tumor and came back to the transducer. Meanwhile, the average Nakagami parameters on and beneath the tumor were 0.8 and 0.66 , respectively, just corresponding to a $17 \%$ decrease in the SNR of the Nakagami image scan line.

To clearly reveal the differences in a Nakagami image corresponding to a cyst, fat and a tumor in the phantom, we compared the values of $\mu_{\bar{m}_{\mathrm{w}}} \pm \mathrm{SD}$, as shown in figure 7 . The result showed that the standard deviations were small for each mass. It indicated that the uniformity of parameter estimation by the proposed method is good under the ideal condition, making the Nakagami image successful at classifying different local breast masses $(p<0.001)$. However, it was evident that the $m_{\mathrm{w}}$ parameters in each mass would typically form a distribution, as shown in figures $3-5$. This is due to the fact that the backscattered statistics in a uniform 

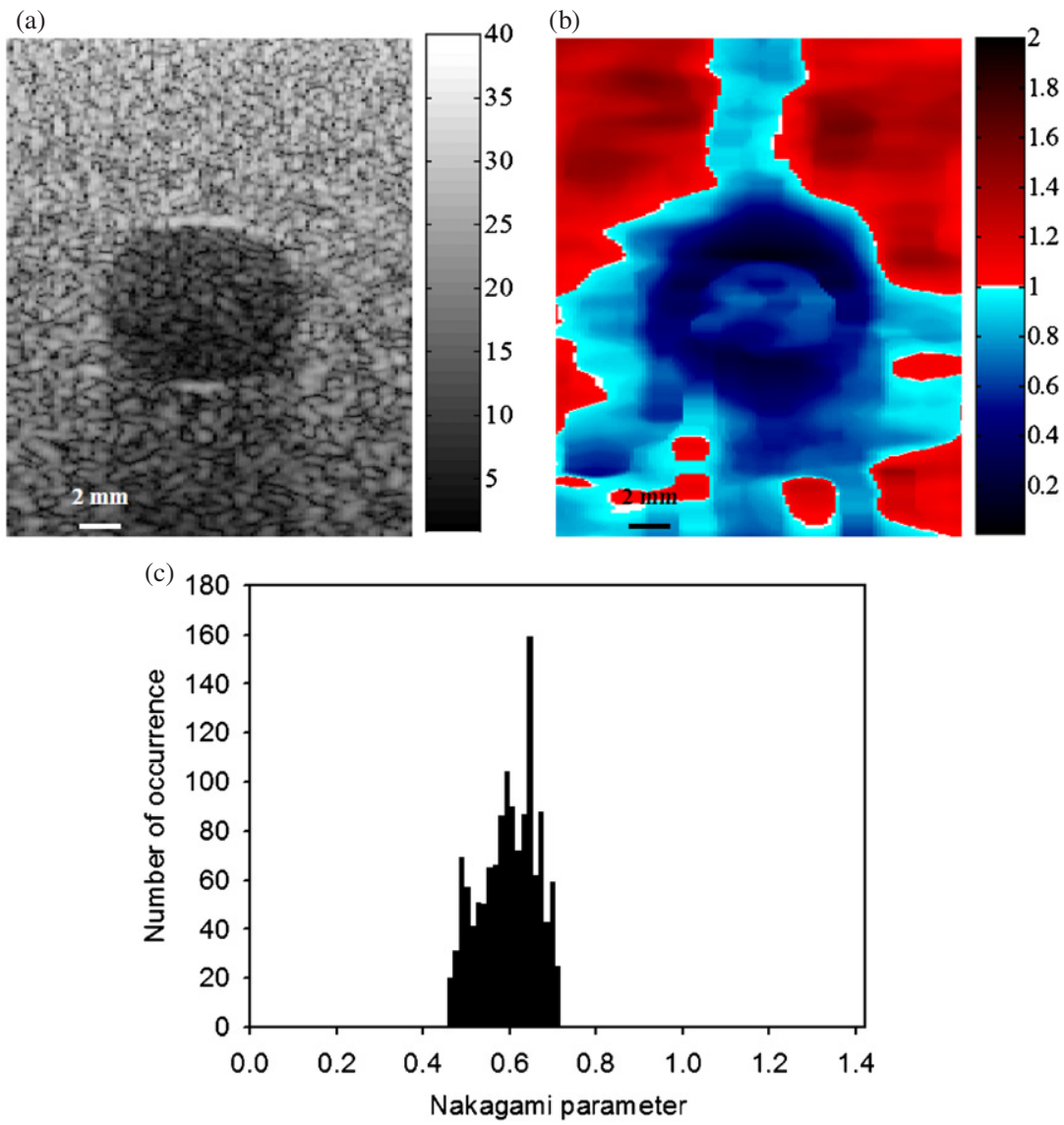

Figure 5. Typical B-mode image (a), Nakagami image (b) and the histogram of the Nakagami image pixels (c) for the tumor.

material would behave locally differently, which has been reported in the previous study (Tsui and Chang 2007, Tsui et al 2007).

Figures 8 (a) and (b) show the B-mode and Nakagami images of the irregular tumor. Figure 8(c) shows the $m_{\mathrm{w}}$ histogram of the irregular tumor. In this case, the Nakagami image showed more blue shading and less red shading inside the tumor, corresponding to the $m_{\mathrm{w}}$ parameters with a distribution ranging from 0.4 to 1.1. Although the $m_{\mathrm{w}}$ distribution in the irregular tumor was wider than that of the regular tumor in figure 5(c), the $\mu_{\bar{m}_{\mathrm{w}}} \pm$ SD value was $0.76 \pm 0.05$, indicating that global statistics of this irregular tumor still belongs to a preRayleigh distribution. It is noteworthy that the tumor contour-which was somewhat unclear due to the shadowing effect in the B-mode image-appeared to be easier to distinguish in the Nakagami image. This is because the Nakagami image, which is just determined by the envelope shape and not influenced by the signal magnitude, would be less sensitive to the attenuation effect. Figure 9 shows that the averages of relative intensities on and beneath the tumor are 0.35 and 0.11 , respectively, indicating a $68 \%$ decrease in the echo SNR. Meantime, the average Nakagami parameters on and beneath the tumor were 0.9 and 1.02 , respectively. It is clear that there was no significant changes in the SNR of the Nakagami scan 


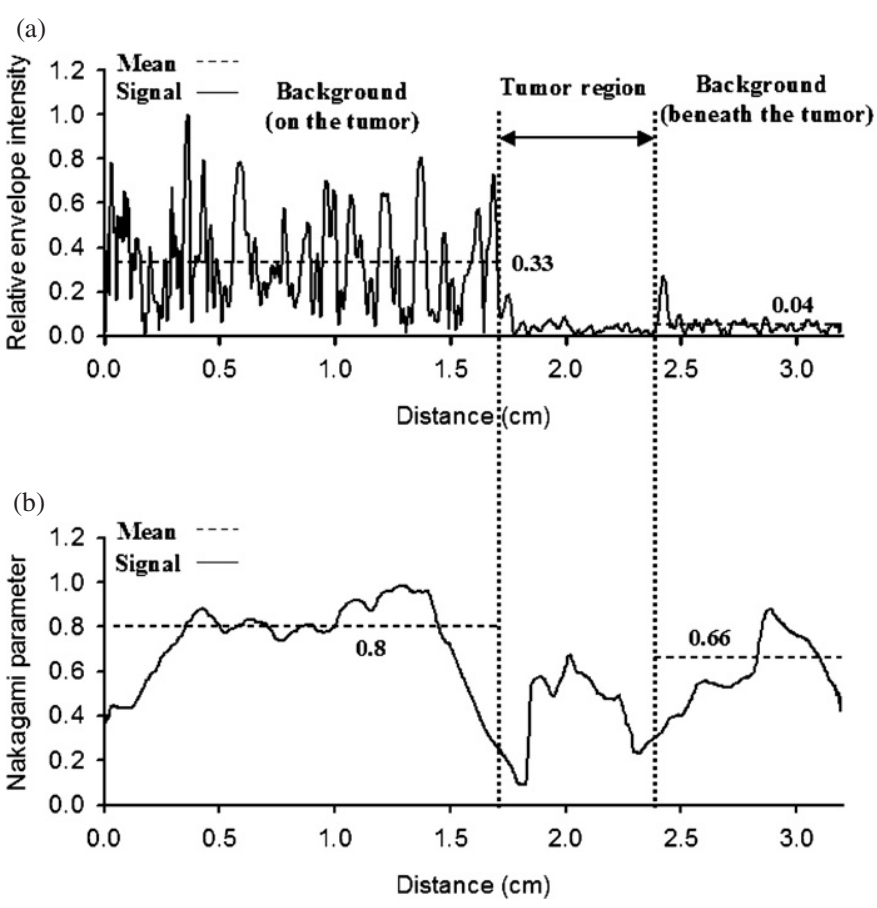

Figure 6. Vertical image profiles (i.e. scan lines) of the B-mode (a) and Nakagami images (b) for the tumor in the phantom.

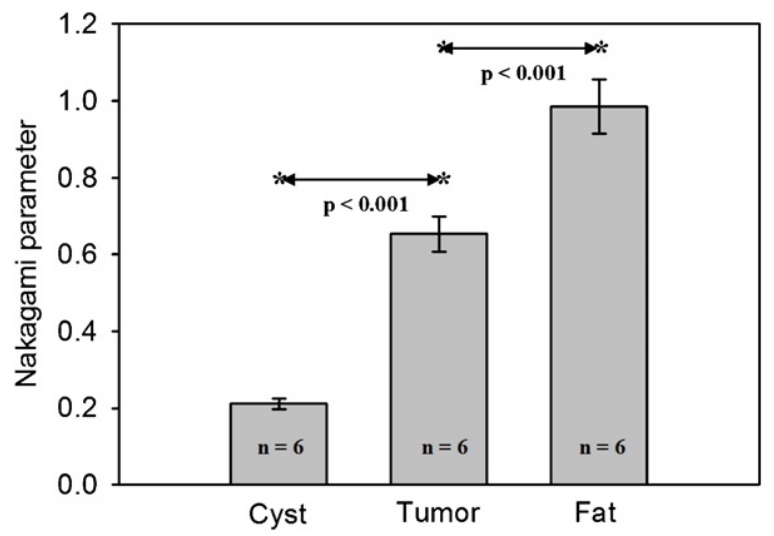

Figure 7. Comparison of Nakagami parameters for the cyst, tumor and fat in the phantom. Data are mean and SD values.

line. Note that the Nakagami parameter beneath the tumor is not necessarily smaller than that on the tumor because it is only determined by the signal shape.

On the other hand, we observe that the tumor contour in the Nakagami image corresponded to very-dark-blue shading, which was due to the so-called subresolvable effect of Nakagami imaging at the interface (Tsui et al 2007). The subresolvable effect is also useful to the identification of tumor contour. Because the interface is smaller than the resolution of the 


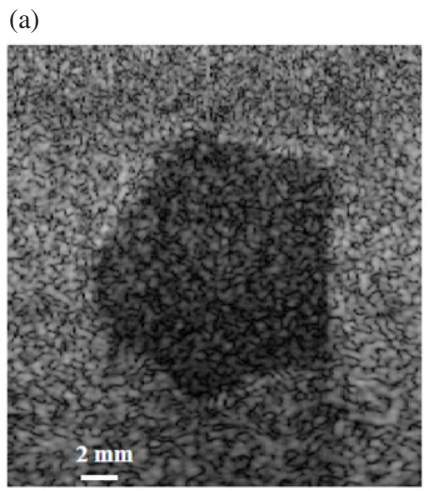

(b)

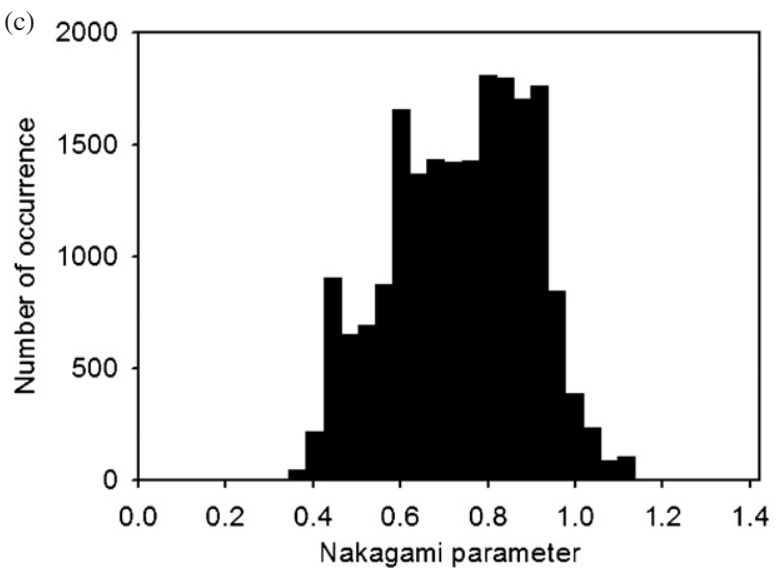

Figure 8. Typical B-mode image (a), Nakagami image (b) and the histogram of the Nakagami image pixels (c) for the irregular tumor.

Nakagami image (i.e. the size of the sliding window), not only echoes from the tumor but also background signals will be acquired when the window moves onto the interface. However, the tumor signal is typically smaller than the background signal; the statistics of the backscattered envelopes collected by the window will tend to conform to a pre-Rayleigh distribution, making the Nakagami parameter very small. We call this the subresolvable effect of Nakagami imaging, which appears at the interface between a tumor and glandular tissue. This effect can make it easier to identify the tumor contour (and hence the tumor-related region) in the Nakagami image than in the conventional B-mode image when the shadowing effect of the tumor is strong. Nevertheless, it should be noted that the subresolvable effect of Nakagami imaging would simultaneously influence the detection of small extensions of the tumor, because the backscattered statistics of tumors smaller than the Nakagami image resolution would shift extremely toward a pre-Rayleigh distribution corresponding to a small Nakagami parameter, which has shading close to that of the interface or cyst. To remedy this weakness, a higher frequency ultrasound may be used to enhance the resolution of Nakagami imaging.

In order to explore the tolerance of the shadowing effect in the Nakagami image, we performed two further experiments on the irregular tumor using a different scan view and imaging settings. The first example was the irregular tumor imaged at a different scanning 


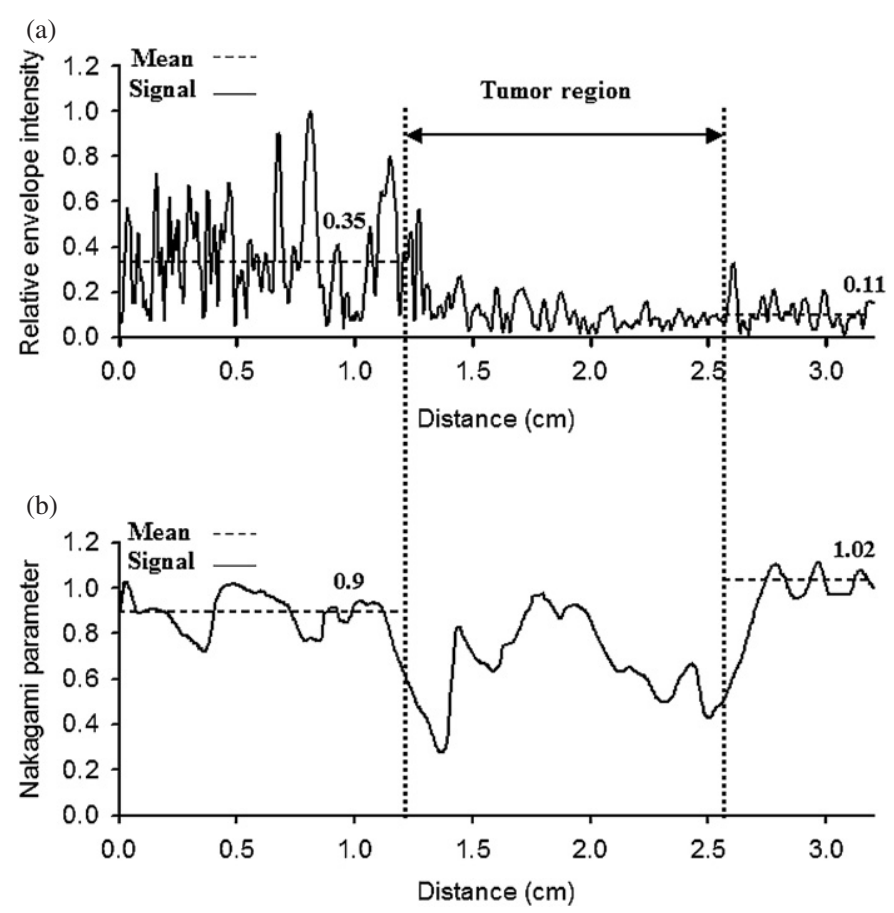

Figure 9. Vertical image profiles (i.e. scan lines) of the B-mode (a) and Nakagami images (b) for the irregular tumor in the phantom.

angle, as shown in figure 10(a). This scan view purposely corresponds to a fraction of the irregular tumor so that the brightness of the tumor in the B-mode scan is close to that of the glandular background. In this case, the tumor contour roughly described by a white dotted line in the B-mode image was too blurred to identify the tumor-related region. In contrast, the Nakagami image clearly showed the tumor contour in very-dark-blue shading caused by the subresolvable effect. The tumor-related region was also identified in the background. The mean \pm standard deviation of the Nakagami image pixels $\left(\bar{m}_{\mathrm{w}} \pm \mathrm{SD}\right)$ in the tumor was $0.63 \pm 0.11$, corresponding to the blue shading and envelope statistics of the pre-Rayleigh distribution. The second example was the irregular tumor imaged at a different scanning angle and a lower system gain, as shown in figure 10(b). In this case, the shadowing effect plus a low system gain resulted in the information on the tumor contour being virtually absent from the B-mode image, with even the breast mass being possibly misinterpreted as a cyst in the glandular tissue. The Nakagami image clearly indicated that this mass was actually a tumor, according to the blue shading with an $\bar{m}_{\mathrm{w}} \pm \mathrm{SD}$ of $0.84 \pm 0.06$ corresponding to pre-Rayleigh statistics. Moreover, the Nakagami image also clarified the location and contour of the tumor. This result shows that the Nakagami image is indeed less affected by the shadowing effect and the system gain when characterizing breast masses. However, it should be noted that in this example, $\bar{m}_{\mathrm{w}}$ was a little larger than the average Nakagami parameter of the tumor in figure 7. This may be due to the fact that the noise effect makes the envelope statistics close to the Rayleigh distribution (Tsui et al 2005). It suggests that the system gain cannot be too small to provide significant backscattering information when using the Nakagami image to characterize tissues. 
(a)

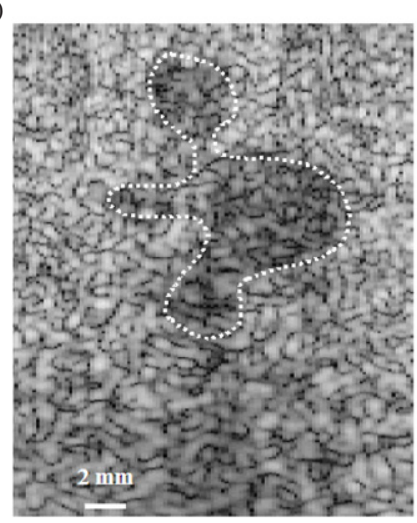

(b)

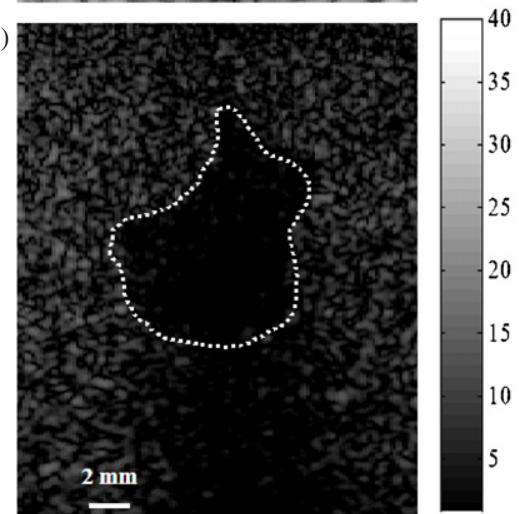

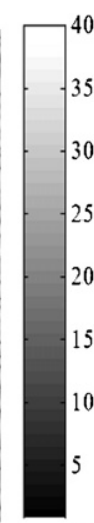
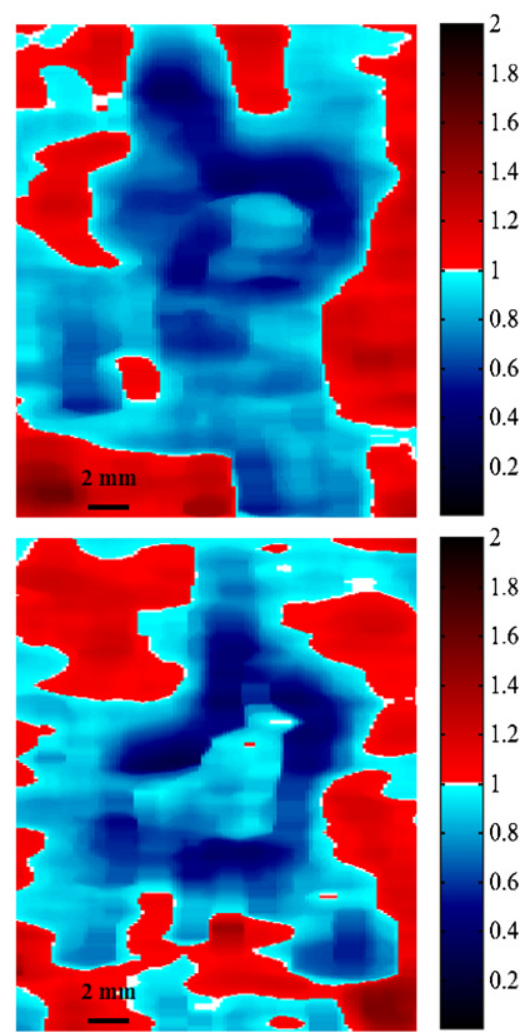

Figure 10. (a) B-mode and Nakagami images of the irregular tumor for a different scanning angle. (b) B-mode and Nakagami images of the irregular tumor for a different scanning angle and a low system gain. The tumor regions are roughly described by white dotted lines.

\subsection{Results on clinical data}

Figure 11 shows the typical B-mode and Nakagami images of breast masses that frequently appear in the clinic. The imaging result of the cyst shown in figure 11(a) agrees well with those obtained in the phantom experiments in figure 3. It was found that $\bar{m}_{\mathrm{w}} \pm \mathrm{SD}$ in the cyst region was $0.2 \pm 0.03$, corresponding to very-deep-blue shading of an extremely pre-Rayleigh distribution. We further collected more cases of cyst $(n=4)$ and calculated the average and standard deviation of the $\bar{m}_{\mathrm{w}}$ value of each case $\left(\mu_{\bar{m}_{\mathrm{w}}} \pm \mathrm{SD}\right) . \quad \mu_{\bar{m}_{\mathrm{w}}} \pm \mathrm{SD}=0.14 \pm 0.03$, indicating that the statistical distribution of the backscattered envelopes from the cyst indeed globally conformed to pre-Rayleigh statistics. This was because the cyst had a very low scatterer concentration.

The typical image of the fat is shown in figure 11(b). Note that some localized fat deposits behaving like masses with lower brightness appeared in the deeper glandular tissue, as indicated by symbol markers in the figure. In this figure, some fat regions were evident in the Nakagami image as red-blue-interlaced shading with an $\bar{m}_{\mathrm{w}} \pm \mathrm{SD}$ of $0.9 \pm 0.08$. Furthermore, $\mu_{\bar{m}_{\mathrm{w}}} \pm \mathrm{SD}=0.89 \pm 0.08(n=6)$, representing the global backscattered statistics of fat close to the Rayleigh distribution. This agrees with the phantom experiments, but differs from that of Raju and Srinivasan (2002) showing that the envelope statistics for fat is a pre-Rayleigh distribution. We consider that this discrepancy is attributable to the previous 

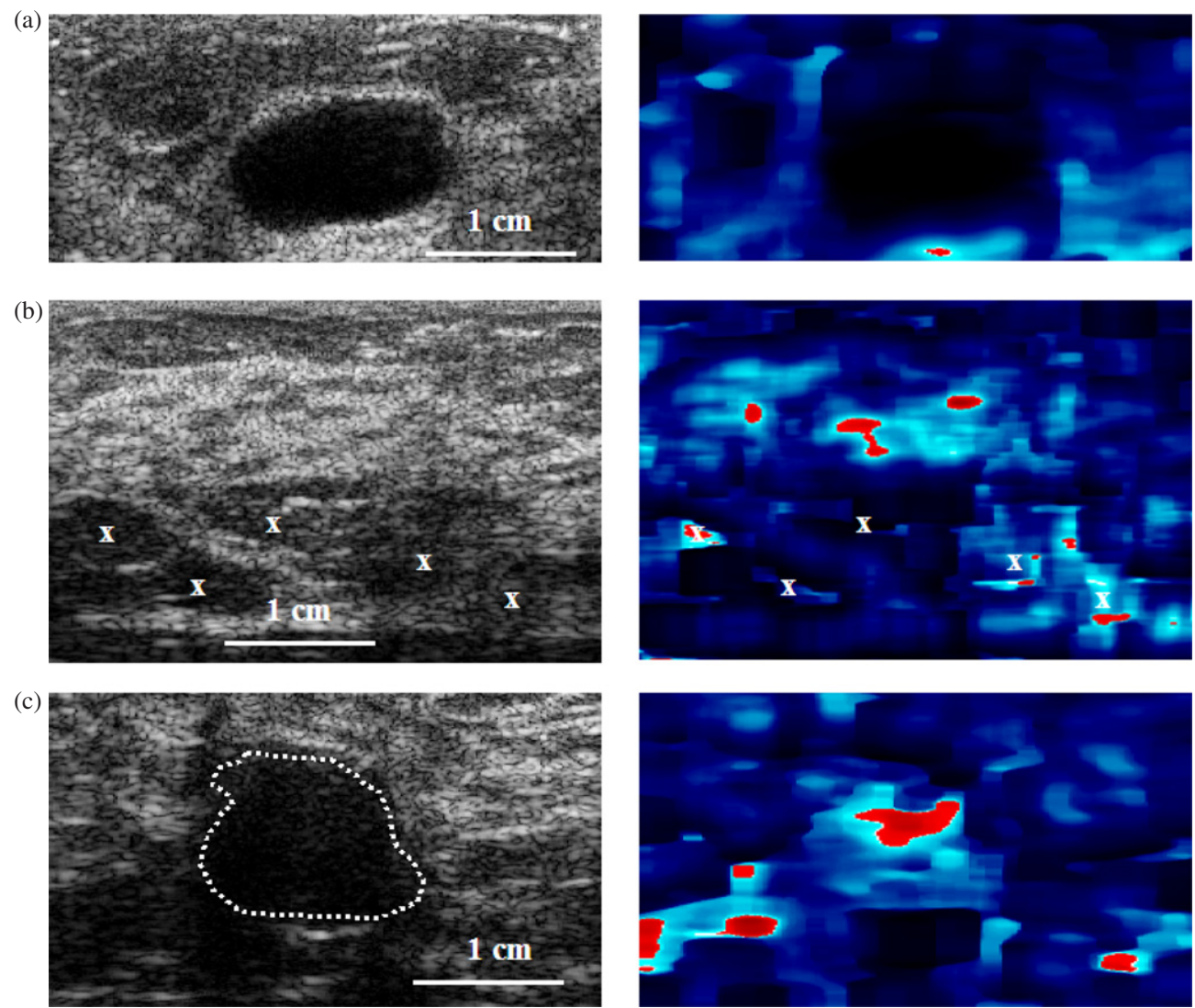

Figure 11. B-mode and Nakagami images of typical clinical breast masses including a cyst (a), fat (b) and a tumor (fibroadenoma) (c). The ' $\mathrm{x}$ ' symbols indicate localized fat deposits. The tumor regions are roughly described by a white dotted line.

study using high-frequency ultrasound (at $28 \mathrm{MHz}$ ). It is well known that the size of the transducer resolution cell varies with the ultrasonic frequency, resulting in different apparent backscattered statistics for the same scatterer structure (Chen et al 1994, Zagzebski et al 1999, Tsui and Wang 2004). On the other hand, we noted that other fat regions were evident in the Nakagami image with only blue shading, corresponding to pre-Rayleigh statistics $\left(\bar{m}_{\mathrm{W}}<1\right)$. This does not agree with the phantom experiments, but this apparent discrepancy might be attributable to these fat regions not being fully developed such that they still contain more glandular tissues, resulting in pre-Rayleigh statistics caused by a higher degree of variability in the scattering cross section.

The typical image of a fibroadenoma is shown in figure 11(c). The tumor region was associated with more blue shading and less red shading corresponding to an $\bar{m}_{\mathrm{w}} \pm$ SD of $0.73 \pm 0.09 . \mu_{\bar{m}_{\mathrm{w}}} \pm \mathrm{SD}=0.67 \pm 0.11(n=8)$. Indeed, the Nakagami image showed that the statistics of backscattered echoes from the tumor conformed to a pre-Rayleigh distribution. This agrees with the findings in phantom experiments. The tumor exhibited backscattered statistics of a pre-Rayleigh distribution for the following reason. Generally speaking, the tumor exhibited a higher sound speed and density, which is due to the formation of a tumor involving a fibrosis process with local glandular tissues in the breast. The local fibrosis in 


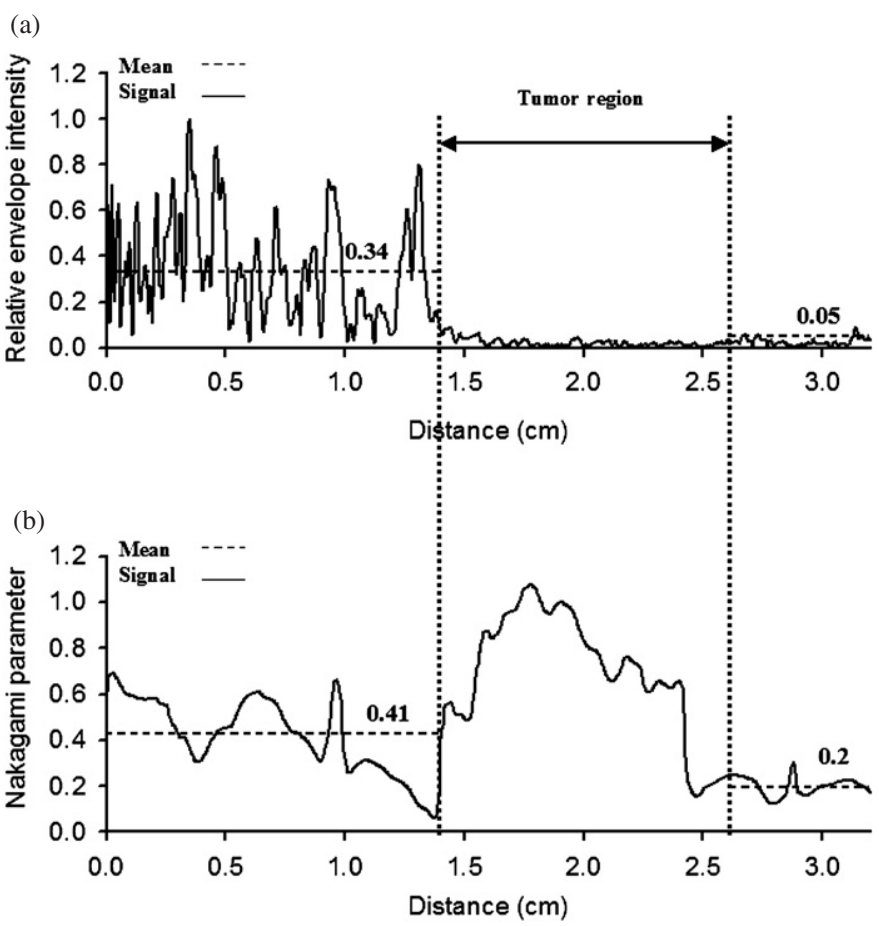

Figure 12. Vertical image profiles (i.e. scan lines) of the B-mode (a) and Nakagami images (b) for the clinical tumor.

the breast changes the properties of the scatterers so as to locally increase the sound speed, density, hardness and echogenicity. Therefore, the scatterers in a tumor exhibit a higher degree of variability in the scattering cross section, resulting in its backscattered statistics approaching a pre-Rayleigh distribution.

In addition, the real tumor tissue exhibited a strong shadowing effect in its B-mode image. In particular, the two sides beneath the tumor had a stronger shadowing effect than the central area. Previous studies have indicated that in most cases, the attenuation is higher in tumors (Harper et al 1983, Sickles et al 1984, Stavros et al 1995). The higher attenuation accordingly causes a strong shadowing effect to prevent a definite description of the contour below the tumor. In contrast, the tumor-related region could be identified by the shading distribution in the corresponding Nakagami image, as shown in figure 11. To confirm this point, we showed the representative scan lines of the B-mode and Nakagami images of the tumor in figure 12. The decrease in the signal SNR of the B-scan was up to $94 \%$. Meanwhile, there was just a $51 \%$ decrease in the signal SNR of the Nakagami image. This demonstrated that the Nakagami image could be largely independent of the attenuation effect, facilitating the extraction of most backscattering information in the tumor.

Finally, the values of $\mu_{\bar{m}_{\mathrm{w}}} \pm$ SD for clinical masses were compared in figure 13 . Indeed, the Nakagami image could complement the conventional B-scan to improve the mass classification in clinic $(p<0.005)$. Nevertheless, the comparison between figures 7 and 13 further shows some differences between the phantom and clinical results. Compared to the phantom results for the ideal condition, the clinical results of the Nakagami parameter from tumors of different cases were larger and closer to those of fat and had a higher standard deviation. The possible reason may be due to the fact that the real tumors have a larger diversity and complexity in 


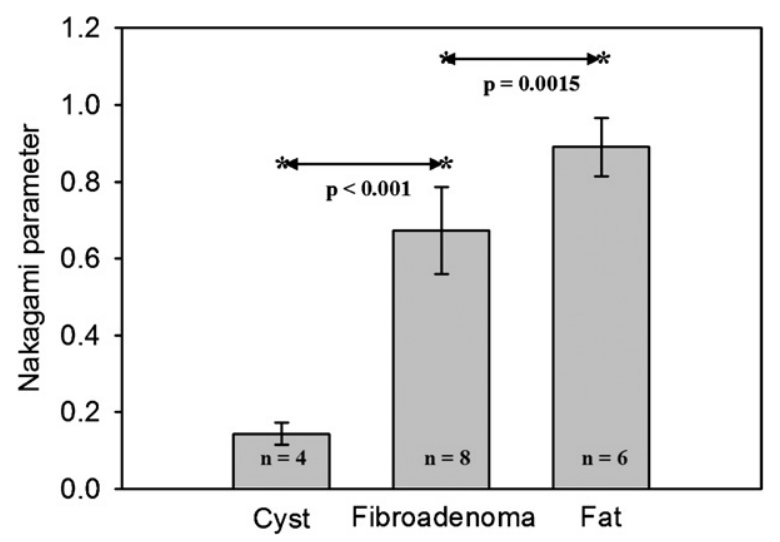

Figure 13. Comparison of Nakagami parameters for the clinical cases of cysts, tumors (fibroadenoma) and fat. Data are mean and SD values.

their properties and structures. The phantom made of uniform materials does not consider the variations in sizes, shapes and echogenicities of scatterers in reality. This is also the reason why the B-scan of the phantom tended to be a more homogeneous speckle image than those of the clinical cases, as shown in figures 3-5, and the average Nakagami parameter in the breast background is smaller than that of the phantom background, as indicated by comparing figures 6,9 and 12 . To better classify different masses, a large-scale clinical validation is required to adequately understand the behaviors of backscattered statistics of different breast lesions including malignant tumors.

\section{Concluding remarks}

We have performed experiments on a breast-mimicking phantom and some typical clinical cases to explore the feasibility of using the Nakagami parametric image to classify breast masses under ideal and practical conditions. The experimental results show that the Nakagami image is not subject to the shadowing effect that frequently occurs beneath high-attenuation masses in conventional B-mode scans. This is due to the estimations of the Nakagami parameter being mainly dependent on the statistics of the backscattered envelope and less affected by the echo amplitude. The advantages of using the Nakagami imaging to characterize breast masses and some differences of the Nakagami results between ideal and practical conditions are as follows. (1) The Nakagami image can visually distinguish different breast masses and local properties, which can avoid the introduction of bias in estimating the Nakagami parameter of the mass when an inappropriate region of interest is chosen to collect envelope data that are not from the mass; (2) the Nakagami image can complement the B-scan to improve describing the mass contour for identifying the tumor-related region when the shadowing effect in the B-mode scan is stronger or a low system gain is used, and thereby reduce the dependence of the diagnostic results on the skill of the operator; and (3) compared to the phantom results for the ideal condition, the clinical results of the Nakagami parameter from tumors of different cases are larger and highly variant, because the real tumors have a larger complexity in their properties and structures of scatterers. For a better mass classification, a large-scale clinical validation is required to thoroughly explore the behaviors of backscattered statistics of different breast lesions including malignant tumors. 


\section{Acknowledgments}

The authors thank Professor P C Li (Department of Electrical Engineering, National Taiwan University, Taipei, Taiwan) for providing the breast phantom and Dr B H Lee (Mackay Memorial Hospital, Hsinchu, Taiwan) for supporting the clinical experiments. This work was supported by Academia Sinica under grant no. AS-98-TP-A02, National Tsing Hua University under grant no. 97N2524E1 and the National Science Council of the Republic of China (Taiwan) under grant no. NSC96-2221-E-002-201.

\section{References}

Alizad A, Whaley D H, Greenleaf J F and Fatemi M 2006 Critical issues in breast imaging by vibro-acoustography Ultrasonics 44 e217-20

Bevan P D and Sherar M D 2001a B-scan ultrasound imaging of thermal coagulation in bovine liver: log envelope slope attenuation mapping Ultrasound Med. Biol 27 379-87

Bevan P D and Sherar M D 2001b B-scan ultrasound imaging of thermal coagulation in bovine liver: frequency shift attenuation mapping Ultrasound Med. Biol 27 809-17

Bridal S L, Fornes P, Bruneval P and Berger G 1997 Parametric (integrated backscatter and attenuation) images constructed using backscattered radio frequency signals $(25-56 \mathrm{MHz})$ from human aortae in vitro Ultrasound Med. Biol. 23 215-29

Chen J F, Zagzebski J A and Madsen E L 1994 Non-Gaussian versus non-Rayleigh statistical properties of ultrasound echo signals IEEE Trans. Ultrason. Ferroelectr. Freq. Control 41 435-40

Davignon F, Deprez J F and Basset O 2005 A parametric imaging approach for the segmentation of ultrasound data Ultrasonics 43 789-801

Drukker K, Giger M L and Mendelson E B 2003 Computerized analysis of shadowing on breast ultrasound for improved lesion detection Med. Phys. 30 1833-42

Dumane V A, Shankar P M, Piccoli C W, Reid J M, Forsberg F and Goldberg B B 2002a Computer aided classification of masses in ultrasonic mammography Med. Phys. 29 1968-73

Dumane V A, Shankar P M, Piccoli C W, Reid J M, Genis V, Forsberg F and Goldberg B B 2002b Classification of ultrasonic B mode images of the breast using frequency diversity and Nakagami statistics IEEE Trans. Ultrason. Ferroelectr. Freq. Control $49664-8$

Glide C, Duric N and Littrup P 2007 Novel approach to evaluating breast density utilizing ultrasound tomography Med. Phys. 34 744-53

Harper P A, Kelly-Fry E, Noe J S, Bies J R and Jackson V P 1983 Ultrasound in the evaluation of solid breast masses Radiology 146 731-6

Holfman H C 1960 Statistical Methods on Radio Wave Propagation (New York: Pergamon)

Huang S W and Li P C 2004 Tomographic reconstruction of sound velocity distribution in the breast using linear arrays: experimental results IEEE Ultrason Symp. Proc. vol 3 pp 1844-7

Huang S W and Li P C 2005 Ultrasonic computed tomography reconstruction of the attenuation coefficient using a linear array IEEE Trans. Ultrason. Ferroelectr. Freq. Control 52 2011-22

Jackson V P 1990 The role of US in breast imaging Radiology 177 305-11

Kolár R, Jirik R and Jan J 2004 Estimator comparison of the Nakagami-m parameter and its application in echocardiography Radioengineering 13 8-12

Kolb T M, Lichy J and Newhouse J H 1998 Occult cancer in women with dense breasts: detection with screening US-diagnostic yield and tumor characteristics Radiology 207 191-9

Kubik-Huch R A 2006 Imaging the young breast Breast 15 S35-40

Ma L, Fishell E, Wright B, Hanna W, Allan S and Boyd N F 1992 Case-control study of factors associated with failure to detect breast cancer by mammography J. Natl Cancer Inst. 84 781-5

Moore S K 2001 Better breast cancer detection IEEE Spectr. 38 50-4

Raju B I and Srinivasan M A 2002 Statistics of envelope of high-frequency ultrasonic backscatter from human skin in vivo IEEE Trans. Ultrason. Ferroelectr. Freq. Control. $49871-82$

Shankar P M 2000 A general statistical model for ultrasonic backscattering from tissues IEEE Trans. Ultrason. Ferroelectr. Freq. Control. 47 727-36

Shankar P M, Dumane V A, Piccoli C W, Reid J M, Forsberg F and Goldberg B B 2002 Classification of breast masses in ultrasonic B-mode images using a compounding technique in the Nakagami distribution domain Ultrasound Med. Biol. 28 1295-300 
Shankar P M, Dumane V A, George T, Piccoli C W, Reid J M, Forsberg F and Goldberg B B 2003a Classification of breast masses in ultrasonic B scans using Nakagami and K distributions Phys. Med. Biol. 48 2229-40

Shankar P M, Dumane V A, Piccoli C W, Reid J M, Forsberg F and Goldberg B B 2003b Computer-aided classification of breast masses in ultrasonic B-scans using a multiparameter approach IEEE Trans. Ultrason. Ferroelectr. Freq. Control 50 1002-9

Shankar P M, Dumane V A, Reid J M, Genis V, Forsberg F, Piccoli C W and Goldberg B B 2001 Classification of ultrasonic B-mode images of breast masses using Nakagami distribution IEEE Trans. Ultrason. Ferroelectr. Freq. Control. 48 569-80

Shankar P M, Piccoli C W, Reid J M, Forsberg F and Goldberg B B 2005 Application of the compound probability density function for characterization of breast masses in ultrasound B scans Phys. Med. Biol. 50 2241-8

Shung K K, Smith M B and Tsui B 1992 Principles of Medical Imaging (San Diego, CA: Academic)

Sickles E A, Filly R A and Callen P W 1984 Benign breast lesions: ultrasound detection and diagnosis Radiology $151467-70$

Stavros A T, Thickman D, Rapp C L, Dennis M A, Parker S H and Sisney G A 1995 Solid breast nodules: use of sonography to distinguish between benign and malignant lesions Radiology 196 123-34

Tsui P H and Chang C C 2007 Imaging local scatterer concentrations by the Nakagami statistical model Ultrasound Med. Biol. 33 608-19

Tsui P H, Huang C C, Chang C C, Wang S H and Shung K K 2007 Feasibility study of using high-frequency ultrasonic Nakagami imaging for characterizing the cataract lens in vitro Phys. Med. Biol. 52 6413-25

Tsui P H and Wang S H 2004 The effect of transducer characteristics on the estimation of Nakagami parameter as a function of scatterer concentration Ultrasound Med. Biol. $301345-53$

Tsui P H, Wang S H, Huang C C and Chiu C Y 2005 Quantitative analysis of noise influence on the detection of scatterer concentration by Nakagami parameter J. Med. Biol. Eng. 25 45-51

Whatmough C, Guitian J, Baines E, Benigni L, Mahoney P N, Mantis P and Lamb C R 2006 Ultrasound image compounding: effect on perceived image quality Vet. Radiol. Ultrasound 48 141-5

Zagzebski J A, Chen J F, Dong F and Wilson T 1999 Intervening attenuation affects first-order statistical properties of ultrasound echo signals IEEE Trans. Ultrason. Ferroelectr. Freq. Control 46 35-40

Zonderland H M 2000 The role of ultrasound in the diagnosis of breast cancer Semin. Ultrasound. CT MR 21 317-24 